\title{
RELEVANCIA SOCIAL, PROFESIONAL Y DISCIPLINAR DEL ABORDAJE DE ENFERMERÍA DE LA SALUD CARDIOVASCULAR EN POBLACIÓN TRABAJADORA
}

\author{
SOCIAL, PROFESSIONAL AND DISCIPLINER RELEVANCE OF NURSING \\ CARDIOVASCULAR HEALTH AMONG WORKERS
}

VIVIAN VÍLCHEZ BARBOZA*

DENIS CARRILLO DÍAZ**

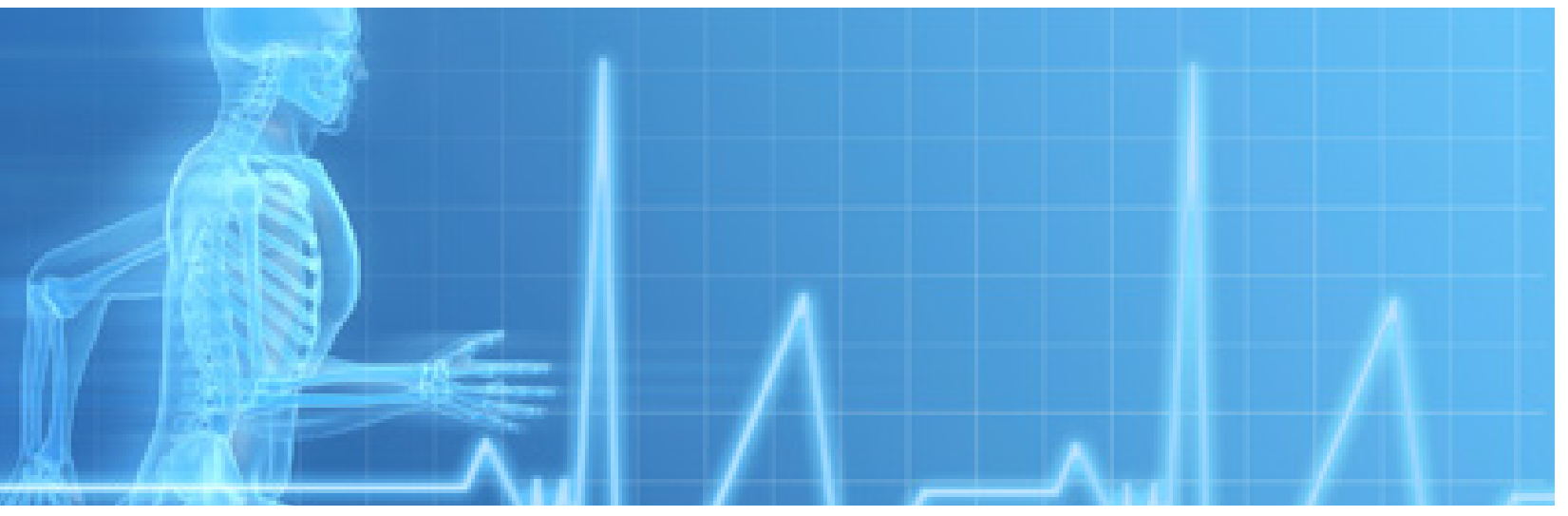

\section{ABSTRACT}

Reflection article, which aims to make a reflection on the social, professional and discipliner relevance to tackle cardiovascular health in the Nursing Intervention in Adult Workers Signature, located in the VII semester of the curriculum of the Nursing career at the University of Costa Rica. It begins with a look at the state of art in Costa Rica, the challenges posed to the health sector cardiovascular diseases and the importance it represents for Nursing incorporate this subject in its curriculum.

Key Words: Cardiovascular Nursing, quality of life, teaching.
(*)Doctora en Enfermería. Docente del Módulo Intervención de Enfermería en la Adultez Sana. Docente-Investigadora Escuela de Enfermería. Universidad de Costa Rica. Correo: vivian.vilchez@ucr.ac.cr

$\left.{ }^{(* *}\right)$ Máster en Enfermería en Enfermería Ginecológica, Obstétrica y Perinatal. Sociólogo. Coordinador del Módulo Intervención de Enfermería en la Adultez Sana. Docente-Investigador Escuela de Enfermería. Universidad de Costa Rica. Correo: dennisc66@gmail.com

\section{RESUMEN:}

E objetivo del presente artículo es efectuar una reflexión sobre la relevancia social, profesional y disciplinar de abordar la salud cardiovascular en población trabajadora, en el Módulo Intervención de Enfermería en la Adultez Sana, ubicado en el VIII semestre del plan de estudios de la carrera de Enfermería de la Universidad de Costa Rica. Se inicia con una mirada sobre el estado del arte en Costa Rica, los retos que representan las enfermedades cardiovasculares al sector salud y la importancia que representa para la Enfermería incorporar esta temática en su plan de estudios.

Palabras Claves: Enfermería Cardiovascular, calidad de vida, enseñanza

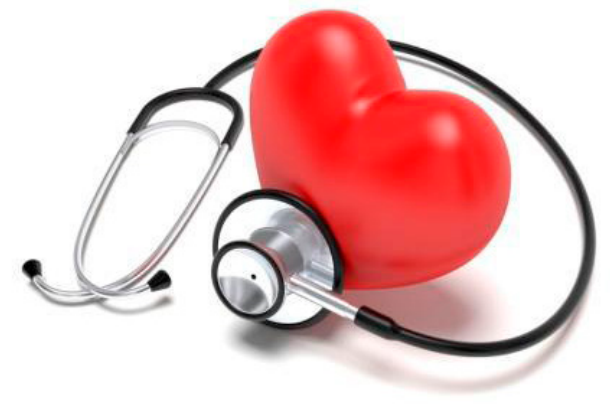




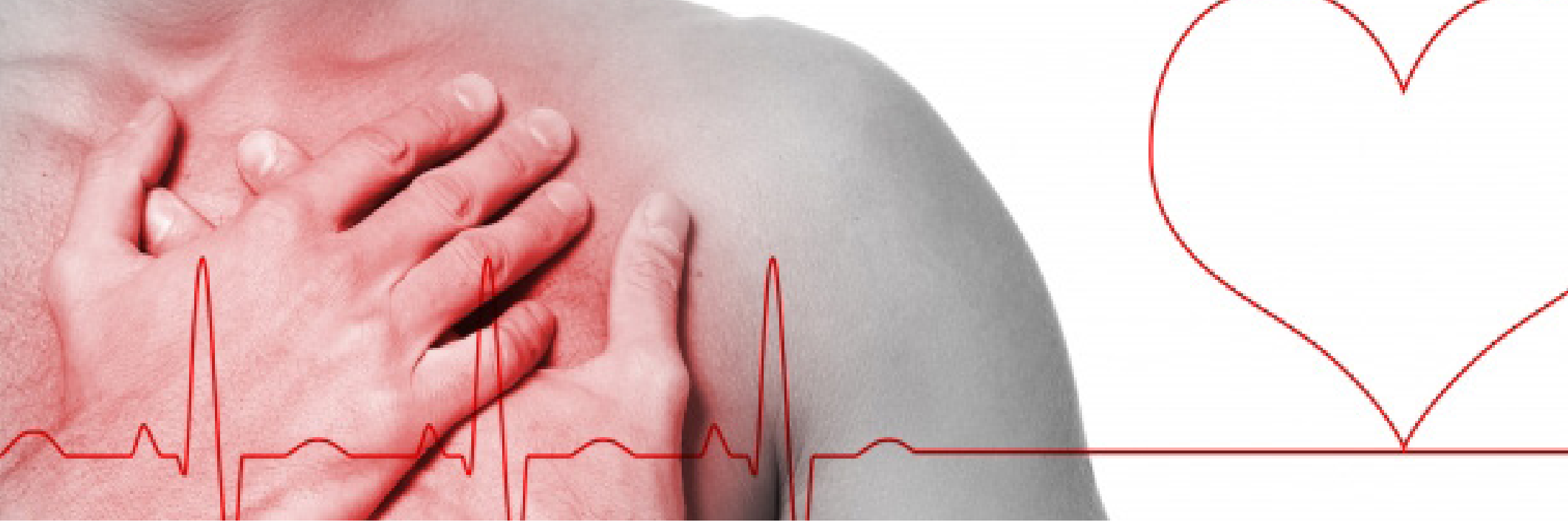

La Organización Mundial de la Salud ${ }^{(1)}$ considera a las enfermedades no transmisibles como la principal causa de mortalidad y discapacidad mundial, constituyendo uno de los mayores retos que enfrentan los sistemas de salud, debido al gran número de casos afectados que conforman la causa más frecuente de incapacidad prematura, la complejidad y costo elevado de su tratamiento. Por tal motivo, se convierte en un problema de emergencia de la salud pública, que se da como resultado de cambios sociales y económicos que modificaron el estilo de vida de un gran porcentaje de la población ${ }^{(2)}$.

Se plantea como enfermedades no transmisibles principales: las enfermedades cardiovasculares, diabetes, cáncery enfermedades respiratorias crónicas ${ }^{(3)}$. En América Latina y el Caribe las Enfermedades no Transmisibles representan $57.9 \%$ de la mortalidad general, entre las cuales figuran: las cardiovasculares con un $45.4 \%{ }^{(4)}$. Las muertes por Enfermedad Cardiovascular (ECV) afectan por igual a ambos sexos, y más del $80 \%$ se producen en países de ingresos bajos y medios. Además, se reconoce el aumento de la enfermedad cardíaca coronaria y la hipertensión arterial como precursores del paro cardiaco; ${ }^{(1)}$ y la identificación de los principales factores de riesgo del comportamiento que contribuyen a la mortalidad por $E C V^{(5)}$ y en los que se deben dirigir las acciones en salud: hipertensión arterial, consumo de tabaco, elevación de la glicemia, inactividad física, sobrepeso y obesidad. Aunado a esto, la Organización Panamericana ${ }^{(3)}$ indica que se puede prevenir hasta el $75 \%$ de la enfermedad isquémica del corazón y de los accidentes cerebrovasculares, si se gestiona el conocimiento científico disponible sobre la prevención y el control de sus factores de riesgo, por medio de intervenciones costo-efectivas y asequibles, que incorporen las vivencias de las personas que las padecen; para lo cual es esencial continuar con investigaciones que incluyan en sus propósitos las mejora del conocimiento de los factores de riesgo cardiovascular y sus determinantes, además de que permitan desarrollar estrategias integrales y sostenibles de educación continuada dirigida especialmente a la atención primaria en salud.
Actualmente, en Costa Rica, la mayor parte de los daños a la salud son producto de las enfermedades del sistema circulatorio y el cáncer. Los indicadores de salud reflejan una transición epidemiológica, que se evidencia no solo en la esperanza de vida de 79.39 años(6) sino también en los cambios de la sociedad, debido a la transición demográfica, el desarrollo tecnológico, la globalización de las comunicaciones $y$ el comercio, lo que ha generado mayor movilidad de las personas y modificaciones en los estilos de vida. Estas modificaciones han favorecido que las principales causas de morbimortalidad; en ambos sexos, sean las enfermedades del sistema circulatorio: infartos y enfermedad isquémica del corazón. Una causa básica de ambas enfermedades es la aterosclerosis (7), la cual es originada por dos tipos de factores de riesgo ${ }^{(8)}$ : los no modificables (edad, sexo, antecedentes familiares de enfermedad cardiovascular) y los modificables o prevenibles (obesidad/ obesidad abdominal, hipertensión arterial, diabetes mellitus, colesterol total y LDL elevados, colesterol $\mathrm{HDL}$ bajo, triglicéridos altos, fumado, sedentarismo, consumo excesivo de alcohol, consumo de anticonceptivos orales, estrés y alimentación no cardioprotectora).

La Caja Costarricense de Seguro Social ( ${ }^{9)}$ (CCSS) señala que en Costa Rica, el $10 \%$ de la población mayor de 20 años padece de hipertensión; 


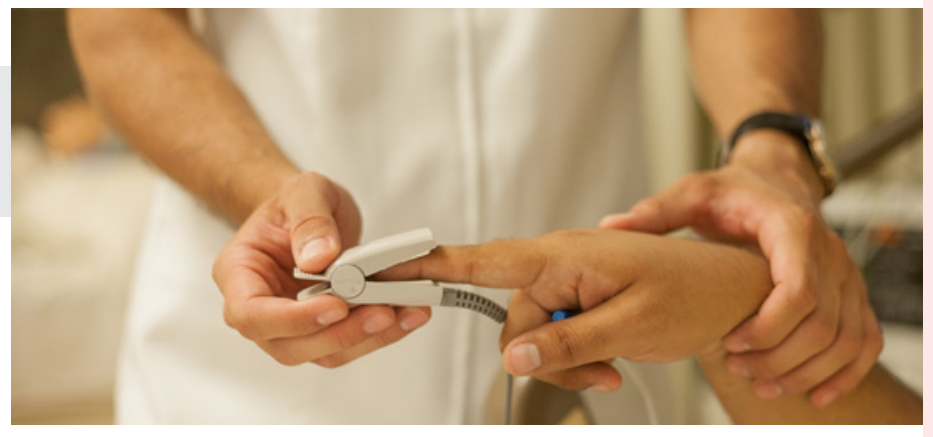

que el $77.3 \%$ de las mujeres entre 45 y 64 años tienen una prevalencia elevada de obesidad (IMC $=30 \mathrm{~kg} / \mathrm{m}^{2}$ ) y diabetes, y el $62.4 \%$ de los hombres entre 20 y 64 años. Los datos sugieren que la prevalencia de sobrepeso y obesidad seguirá incrementándose en los próximos años. ${ }^{(9)}$ Lo mencionado, exige respuestas oportunas y eficaces a las necesidades de salud que producen, las enfermedades cardiovasculares, ya que producen déficit en la calidad de vida de la población. ${ }^{(10)}$

Otro aspecto a considerar, señalado por la literatura, es que los factores de riesgo cardiovascular se asocian con las condiciones de trabajo(11). Desde el origen del ser humano, surgió el trabajo como una necesidad para sobrevivir al medio como una influencia positiva respecto a la salud que produce bienestar, pero puede convertirse también en una influencia potencialmente negativa ${ }^{(12),}$ ya que puede tener efectos sobre la salud física de los/as trabajadores, a través de activaciones hormonales $y$ estimulaciones nerviosas que provocan enfermedades crónicas del sistema locomotor, del respiratorio y del cardiovascular, así como trastornos del comportamiento. ${ }^{(13)}$

La importancia de caracterizar de forma precisa los factores de riesgo cardiovascular en grupos poblacionales específicos, como es el caso de población trabajadora; responde al fin de implantar campañas educativas y protocolos de intervención capaces de optimizar la aplicación de los recursos existentes en salud. ${ }^{14}$ Aunado a lo anterior, los servicios de salud laboral de las empresas o instituciones, constituyen un recurso excelente por la cercanía y accesibilidad para el/la trabajador/a, el cual, es infrautilizado para la detección, seguimiento y control de los factores de riesgo cardiovascular por lo que deberían coordinarse para intervenir más eficientemente en los problemas de salud de la población trabajadora ${ }^{(15)}$.

De acuerdo al contexto presentado, este documento tiene por objetivo efectuar una reflexión sobre la relevancia social, profesional y disciplinar de abordar la salud cardiovascular en población trabajadora, en el Módulo Intervención de Enfermería en la Adultez Sana, en el VII semestre plan de estudios de la carrera de Enfermería de la Universidad de Costa Rica. Inicia con una mirada sobre el estado del arte en el país, los retos que representan las enfermedades cardiovasculares al sector salud y la importancia que representa para la Enfermería incorporar esta temática en su plan de estudios.

1. Es la entidad costarricense encargada de proporcionar los servicios de salud en forma integral a la población habitante de Costa Rica. Asimismo, otorga la protección económica, social y de pensiones.

\section{EXPERIENCIA ACADÉMICA EN EL ABORDAJE DE LA SALUD CARDIOVASCULAR}

Considerando el contexto presentado y acorde a lo propuesto por el $\mathrm{CIE}^{16}$, en cuanto a la transformación de los programas de enseñanza de enfermería para que se centren más en la continuidad asistencial, que engloba la promoción de la salud y la prevención de enfermedades; es que se hizo necesario, este año 2015, introducir una unidad de salud cardiovascular en el Módulo: Intervención de Enfermería con la Adultez Sana, con el objetivo de analizar la salud cardiovascular de los/as trabajadores/as.

Este módulo está ubicado en el VII semestre del plan de estudios de la carrera Licenciatura en Enfermería de la Universidad de Costa Rica. Uno de los propósitos de dicho Módulo, es ofrecer estrategias didácticas para que el estudiantado adquiera conocimientos teórico-prácticos que le faciliten realizar un cuidado de enfermería integral con las personas adultas trabajadoras, en busca de un entorno laboral saludable que favorezca los factores 
protectores y disminuya los factores de riesgos que ponen en peligro la salud de la población; para lo cual, los/as estudiantes cuentan con 16 semanas de práctica y 16 semanas de teoría.

Por lo tanto, para responder a la situación de salud nacional, es que se hace necesario incorporar la unidad de salud cardiovascular, en este Módulo; fundamentada en una relevancia social, profesional y disciplinar.

\section{Relevancia Social:}

Se reporta en Costa Rica desde el año 2000, que las enfermedades cardiovasculares afectan cada vez más, en edades (20 a 64 años) donde se ubica la población económicamente activa. ${ }^{(17)}$ Además existe una importante prevalencia de costarricenses con riesgo cardiovascular moderado/elevado, lo que podría indicar que en 10 años habrá una alta incidencia de casos con eventos cardiovasculares. (18) Es por esta razón, que se considera importante conceptualizar la salud como un producto social, es decir, que la manifestación social se expresa en la estructura biológica; por lo tanto, la salud parte de las experiencias, la interpretación y los problemas planteados por la población. (19) Se comprende que los factores de riesgo y estilo de vida no son agentes

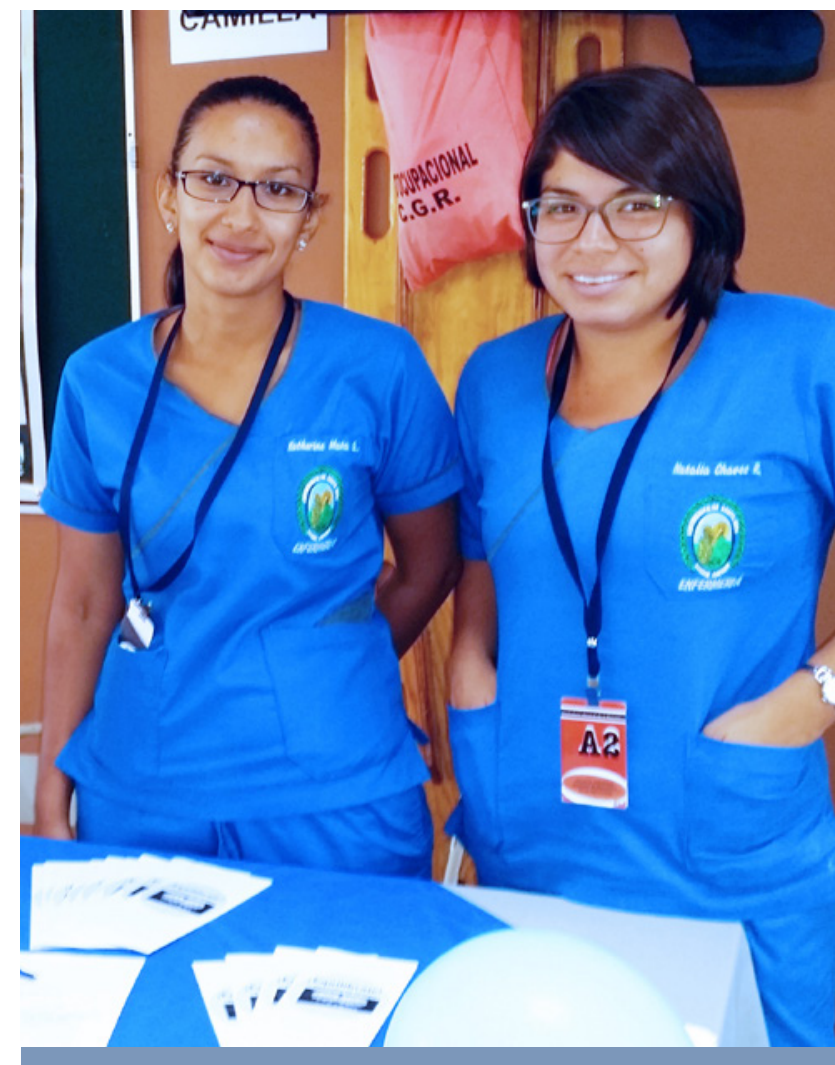

exclusivos causales de enfermedad, ni están descontextualizados de la realidad y del contexto socioeconómico y político; motivo por el cual, surgen los determinantes sociales de la salud, los cuales destacan el reconocimiento de los distintos niveles de determinación social y explican la producción social y la generación de desigualdades e inequidades en salud; ya que las características individuales de las personas y su contexto inmediato no poseen la propiedad de explicar esto. ${ }^{(20)}$

En concordancia con lo expuesto, es que la rotación en un entorno laboral permite al estudiante de Enfermería intervenir desde la promoción de la salud:

- Incentivando y fomentando los factores psicosociales (autoeficacia, apoyo social, resiliencia, optimismo, vitalidad, flexibilidad emocional, espiritualidad, entre otros), los cuales han sido estudiados por su efecto positivo en la salud, al asociarse a menor riesgo de enfermedad cardiovascular de tipo isquémico. ${ }^{(21,22)}$

- Incorporando en las intervenciones, el contexto de la persona, el cual contempla los determinantes sociales de la salud. El/la estudiante desarrolla habilidades y conocimiento para comprender que la salud está relacionada con: a) la capacidad de enfrentar/resolver las situaciones que se presentan en el diario vivir, tanto en el hacer como en el saber; b) las capacidades $y$ las habilidades básicas que no requieren entrenamiento, como comer, dormir, caminar, vestirse, etc, que en algunos momentos de la vida pueden verse alterados; c) una perspectiva subjetiva, sensaciones y emociones que se manifiestan de forma visible en el cuerpo: gestos, ritmo cardiaco, tono de voz, etc.; lo que repercute en los sentimientos y los valores expresados en la vivencia de la salud. (23) 


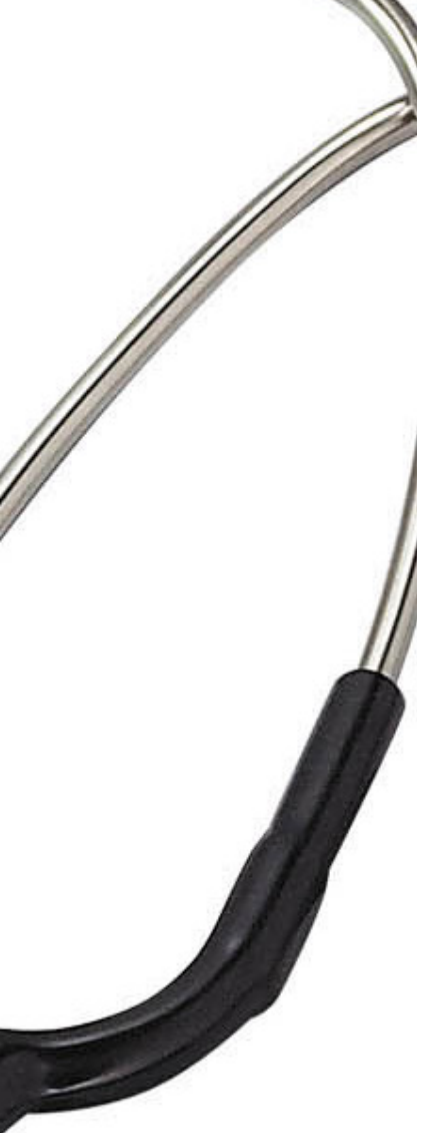



- Impulsando que la persona se apropie de las fortalezas $y$ las herramientas con las que cuenta, para que tome el control sobre su calidad de vida relacionada con salud.

También, el/la estudiante interviene en prevención de la enfermedad, porque encamina acciones a la reducción de factores de riesgo que incidan en manifestaciones del deterioro de la salud al corto, mediano y largo plazo.

\section{Relevancia Profesional:}

Se entiende por salud cardiovascular, como "La capacidad de funcionar adecuadamente, mediante la práctica de actividades que las personas realizan por sí mismas para el mantenimiento del bienestar y la vida".(24)

En este ámbito de la salud, se pretende que el/la estudiante obtenga los conocimientos para vencer el desafío y establecer la prioridad, de detectar los principales factores de riesgo cardiovascular en personas aparentemente sanas, con el propósito de establecer estrategias de cuidado encaminadas a mejorar los estilos de vida en población que aún no presenta daños a la salud(25) a través de un autocuidado responsable, expresado en una dieta sana, en bienestar emocional y en la práctica de ejercicio físico regular. ${ }^{(26)}$
La detección oportuna de los factores de riesgo, es un aspecto de vital importancia, existe evidencia científica que señala que el/la profesional de enfermería debe desarrollar todo su potencial para que pueda implementar intervenciones de enfermería adecuadas a las necesidades de los/as usuarios/as, en este caso mujeres y hombres trabajadores, que contribuyan eficazmente a reducir la carga de las enfermedades cardiovasculares. ${ }^{(16)}$

Los diagnósticos de Enfermería que detectan los/as estudiantes junto con los/as docentes en las personas adultas trabajadoras del sector público en Costa Rica, se enfocan a la promoción de la salud cardiovascular y a prevención de factores de riesgo cardiovascular. Algunos ejemplos según taxonomía NANDA-I ${ }^{(27)}$, son los siguientes:

- (00162) Disposición para mejorar la gestión de la propia salud manifestado por las elecciones de la vida diaria apropiadas para satisfacer objetivos

- (00182) Disposición para mejorar el autocuidado manifestado por expresar deseos de aumentar la responsabilidad en el autocuidado

- (00168) Estilo de vida sedentario relacionado con falta de recursos como lo es el tiempo, manifestado por la elección de una rutina diaria con falta de ejercicio físico y falta de condición física.

- (00001) Desequilibrio nutricional: ingesta superior a las necesidades relacionado con aporte excesivo con relación a la actividad física (gasto Calórico) manifestado por: comer en respuesta a claves internas distintas al hambre (como la ansiedad), patrones de alimentación disfuncional y por estilo de vida sedentario.

- (00055) Desempeño ineficaz del rol manifestado por sobrecarga en el desempeño del rol relacionado con demandas del horario laboral.

- (00177) Estrés por sobrecarga relacionado con la coexistencia de múltiples agentes estresantes manifestado por expresión de exceso de estrés situacional

- (00193) Descuido personal relacionado con 
opción de estilo de vida manifestado por no adherencia a las actividades relacionadas con salud.

Se destaca la importancia de que Enfermería muestre necesidades de atención bajo una mirada integral, que al valorar, diagnosticar, planificar, ejecutar acciones y evaluarlas, evidencie la comprensión de los diversos entornos de las personas (familiar, social, laboral) e incorpore en el análisis de situaciones de salud, el género, para explicar las diferencias que circunscriben el proceso salud-enfermedad cardiovascular en hombres y mujeres. Esto le permite al estudiante, gestionar la movilización de recursos necesarios que favorezcan la salud y el trabajo en red con otras disciplinas y otros sectores.

Otro aspecto, que el/la Enfermero/a deben considerar cuando laboran en el ámbito de la atención primaria, es la necesidad de conocer y mejorar la Calidad de Vida Relacionada con Salud (CVRS), ya que no es suficiente con controlar únicamente variables de morbimortalidad. ${ }^{(28)}$

La CVRS, es un constructo que se estudia desde la década de los 90; se introdujo gradualmente en los estudios de intervención terapéutica y se incorporaron formas de medición ${ }^{(29)}$ de dos maneras: 1) en la práctica clínica para estudiar efectos de intervención terapéutica, tratamiento administrado y la repercusión de los efectos de un programa en las dimensiones de su estado de salud y 2) poblacionales, para estudiar población general o con enfermedades específicas, con el fin de planificar, asignar recursos, priorizar, evaluar políticas de salud, comparar poblaciones. ${ }^{(30)}$ Dichas mediciones representan uno de los avances más importantes en materia de evaluaciones en salud; la cual permite detectar alteraciones e intervenir precozmente, así como comparar las distintas opciones terapéuticas y centrarse de una manera importante en la persona y no en la enfermedad. ${ }^{(31)}$

La importancia de utilizar las mediciones de CVRS, radica, en la contribución en la toma de decisiones que realiza el personal de salud, al incorporar la percepción de los usuarios/as, apoyada en una profunda evidencia empírica de base científica, que considere, además de los indicadores clásicos cuantitativos y los costos, los indicadores cualitativos que expresan el impacto en la calidad de vida y la satisfacción de la persona. ${ }^{(32)}$
Desde el punto de vista subjetivo, CVRS se define como la valoración que realiza una persona, de acuerdo con sus propios criterios del estado físico, emocional $y$ social en que se encuentra en un momento dado, y refleja el grado de satisfacción con una situación personal, a nivel fisiológico, emocional, y social. ${ }^{(32)}$

Existe evidencia sobre la medición de los factores de riesgo cardiovascular a través de los niveles de colesterol, la presión sistólica, el índice de masa corporal, la dieta y la actividad física y además estatus funcional con cuestionario el SF-36 de calidad de vida relacionada con salud. ${ }^{33,} 34$.

Se muestra a continuación un extracto de una nota de enfermería desarrollada por uno de los estudiantes de este Módulo, donde incorpora lo mencionado anteriormente:

MMD es una mujer de 35 años, costarricense, divorciada desde hace 10 años, actualmente con pareja. Se encuentra trabajando en una Institución Pública, desempeñando el puesto de Secretaria. Actualmente está estudiando la carrera de administración de negocios. Se le aplica cuestionario SF-36 para medir su Calidad de Vida Relacionada con Salud (CVRS). En dicha medición, se obtuvieron los siguientes resultados: en el componente de salud física MMD obtiene un puntaje de 64 , el cual indica una percepción algo baja de dicho componente. La dimensión que más alto puntaje obtuvo fue la función física (100) seguido del rol físico (85), esta última debido a que durante las últimas 4 semanas casi siempre ha reducido el tiempo que dedicaba al trabajo $u$ otras actividades a causa de su salud física; principalmente por unas crisis migrañosas que le acontecen mensualmente. En el hospital le realizaron un examen de senografía duppler de arterias cerebrales. Actualmente tiene prescrito migradorixina (ergotamina tartrato $1 \mathrm{mg}$ y clinixinato de 
lisina $125 \mathrm{mg}$ ) y Epival (Valproato semisódico 250 mg). No obstante, el dolor corporal y la salud general fueron las dimensiones con los puntajes más bajos (40 y 30 respectivamente), a causa de que durante las últimas 4 semana ha presentado un dolor muy severo por motivo de un pólipo endometrial diagnosticado, por lo que presenta ciclos menstruales irregulares y con mucho dolor, refiere que este mes su período llegó tres veces seguidas. Se encuentra en control con Ginecología hace 6 meses en el Hospital. Además, considera que se enferma más que las demás personas y que sus defensa disminuyen muy rápido (resfríos constantes). Cuando se divorció entró en una depresión y lleva 10 años tomando 2 tabletas de fluoxetina, además de 2 tabletas de propanolol al sufrir de taquicardia por ansiedad. Esta semana decidió dejar de tomar la fluoxetina, pero ante el estrés de los exámenes universitarios decidió volver a tomarla por un poco más de tiempo.

En el componente de salud mental, la percepción es regular ${ }^{(81)}$, las dimensiones con mayor puntaje en este componente fueron la vitalidad (100) y la salud mental (92) ya que manifiesta sentirse un poco nerviosa por los exámenes universitarios. Seguidamente, la dimensión de función social se encuentra con un puntaje de 70 , por motivo de que durante las últimas 4 semanas algunas veces sus problemas físicos y emocionales previamente descritos han dificultado sus actividades sociales (como visitar amigos, parientes, etc.). La dimensión con el puntaje más bajo fue la de rol emocional (60), esto porque en las últimas 4 semanas algunas veces ha reducido el tiempo que dedicaba al trabajo o a logrado hacer menos de lo que le hubiera gustado, esto por motivo de que el 25 de mayo su hijo de 15 años le escribió un mensaje de texto donde se despedía de ella, en otras palabras intentó suicidarse por una muchacha del colegio que le rechazó. Actualmente se encuentra en control en psiquiatría.

En cuanto a la valoración física, presenta los signos vitales dentro de los parámetros normales: presión arterial de $110 / 70 \mathrm{mmHg}$, frecuencia cardíaca de 66 latidos por minuto, temperatura corporal de $36.4 \mathrm{C}^{\circ}$. Las medidas antropométricas son las siguientes: peso de 72.6 kilogramos y una talla de 1.54 metros, por lo tanto un IMC de 30.6 Una circunferencia de cintura de $89 \mathrm{~cm}$.

El trabajo de Enfermería en la atención primaria en salud, debe enfocarse a que las personas tengan un desarrollo más pleno, que permita el uso de las capacidades físicas y mentales para vivir saludablemente, es decir, aportar más salud a los años. ${ }^{(35)}$

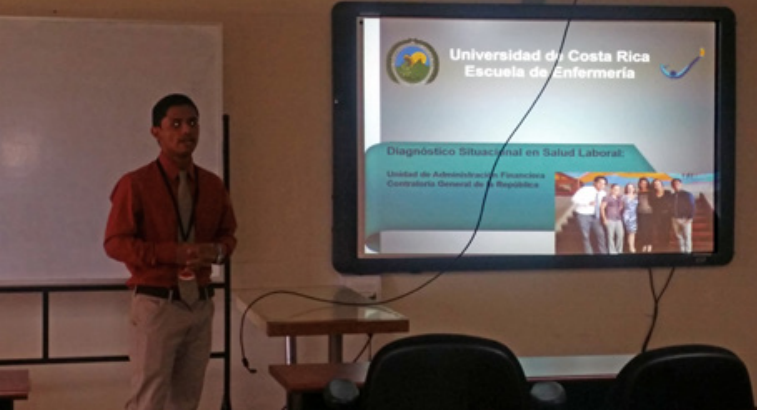

\section{Relevancia Disciplinar:}

Este Módulo encamina sus estrategias didácticas a la unión entre la teoría y la práctica; fomenta el ejercicio de la disciplina implementando el Sistema de Conocimiento en Enfermería Conceptual-Teórico-Empírico (sistema C-T-E), establecido por Fawcett ${ }^{(36)}$, el cual se define como el servicio que se brinda a la sociedad guiado por el conocimiento específico de la disciplina de Enfermería, al articularse con las teorías de la misma.

El paradigma que rige al plan de estudios de la carrera de Enfermería de la Universidad de Costa Rica, es el de integración ${ }^{(37)}$ o visión interacción recíproca 0 interactiva integrativa ${ }^{(38)}$. Este paradigma está representado por el organicismo como forma de interpretar el mundo y los seres caracterizados por el cambio, la persistencia y la interacción. Desde el punto de vista ontológico adopta los Meta paradigmas: persona (es un todo formado por la suma de las partes que están interrelacionadas entre sí y es cambiante de acuerdo a las situaciones que enfrenta, lo que impacta en procesos de salud); entorno (constituido por los diversos contextos en los que vive la persona); salud (pierde su subordinación a la enfermedad; desde esta visión la salud y la enfermedad son dos entidades distintas que coexisten e interaccionan de manera dinámica); cuidado (implica un conocimiento propio de cada persona, que reconoce actitudes, aptitudes, motivaciones y conocimientos) y Enfermería (brindar atención a las personas de una manera integral, donde se reconoce la importancia de lo físico, psicológico, social y espiritual). $Y$ desde el punto de vista epistemológico, esta visión desarrolla el conocimiento a través de abordajes cualitativos y cuantitativos que se complementan y aportan resultados científicos para fundamentar el cuidado.(39) 
Conocer los Meta paradigmas y establecer la visión filosófica, le permite al enfermero/a y en este caso al estudiante; establecer de manera clara la forma de interacción, las intervenciones y los cuidados que Enfermería ofrece.

Asimismo, el/la estudiante utiliza modelos conceptuales como el Modelo de Autocuidado de Dorothea Orem ${ }^{(40)}$ y Teorías como: la de Consecución de Objetivos de Imogene King ${ }^{(40)}$ y la Teoría de las relaciones interpersonales de Hidegard E. Peplau ${ }^{(40)}$. Además emplea para la medición, indicadores empíricos, como: la entrevista del Historial de Enfermería, el Examen Físico, el cuestionario SF-36 Calidad de Vida Relacionada con Salud, entre otros.

De acuerdo con lo anterior, Enfermería debe brindar respuestas a los/as trabajadores/as desde la contribución de un modelo de atención proactivo, enfocado a la salud poblacional, buscando resultados en el ámbito de las mejoras en la calidad de vida; fundamentado en la evidencia científica, para dar soporte al cuidado y una mayor participación en el control de la salud por parte de las personas.

\section{CONSIDERACIONES}

\section{FINALES}

La incorporación de una Unidad de Salud Cardiovascular en el Módulo de Intervención de Enfermería en la Adultez Sana, en el VII semestre, del plan de estudios de la carrera de Enfermería de la Universidad de Costa Rica; responde a una necesidad de salud del país. Brinda respuesta con la implementación de estrategias innovadoras que cumplen con el compromiso que compete a Enfermería, cuidar a una sociedad en transformación permanente, que demanda como docentes, la conducción de procesos pedagógicos consistentes para mediar con el estudiantado diferentes formas de abordaje a los grupos poblacionales desde una postura crítica, humanística y transformadora. Donde se requiere de un accionar de Enfermería, que considere a las personas como sujetos activos y participes de su cuidado, con quienes se establezcan alianzas y acuerdos, desdibujando modelos asistenciales verticales y hegemónicos, aún vigentes.

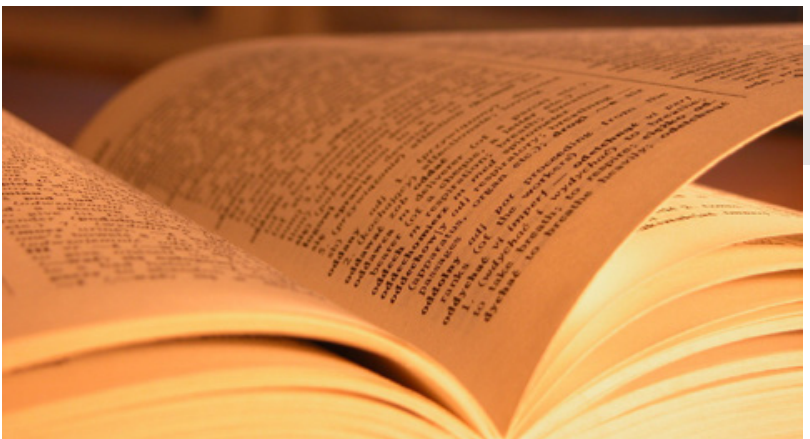

\section{Agradecimiento}

A docentes y estudiantes del Módulo Intervención de Enfermería en la Adultez Sana. I semestre 2015.

\section{REFERENCIAS}

1. Organización Mundial de la Salud. Global status report on noncommunicable diseases. Edición 2010. Geneva: WHO; 2011.

2. Jova R, Rodríguez A, Díaz A, Balcindes S, Sosa I, De Vos $P$ et al. Modelos de atención a pacientes con enfermedades crónicas no transmisibles en Cuba y el mundo. MEDISAN. 2011; 15(11):1609-20.

3. Organización Panamericana de la Salud. La renovación de la atención primaria de la salud en las Américas. Washington D.C.: OPS; 2007. (Documento de Posición de la OPS/OMS).

4. Organización Mundial de la Salud (OMS). Informe sobre la salud en el mundo 2008. La atención primaria de salud más necesaria que nunca. Ginebra [Suiza]: OMS; 2008.

5. Rodríguez-Artalejo F, Banegas JR, Guallar-Castillón P. Epidemiology of Heart Failure. Rev Esp Cardiol. 2004; 57(2):163-70.

6. Instituto Nacional de Estadística y Censo (INEC). [Página principal en Internet], Costa Rica: INEC, Estadísticas Población y Demografía; 2015 [actualizada en agosto 2015; acceso 17 agosto 2015]. http:// www.inec.go.cr/Web/Home/pagPrincipal.aspx.

7. Roldán C, Campos C, Segura de la Morera J, Ruilope LM Evaluación del riesgo cardiovascular y nuevos factores de riesgos de aterosclerosis. Hipertensión. 2005; 22(5): 195-203.

8. Escobar M, Obreque A. Implementación del enfoque de riesgo en el Programa de Salud Cardiovascular. Santiago [Chile]: Ministerio de Salud Chile; 2010.

9. Consejo de Ministros de Salud del Sistema de la Integración Centroamericana (COMISCA). Plan de Salud de Centroamérica y República Dominicana 2010 - 2015. San José [Costa Rica]: COMISCA; 2009.

10. Achury DM, Rodríguez-Colmenares SM, Agudelo-Contreras LA, Hoyos-Segura JR, Acuña-Español JA. Calidad de vida del paciente con enfermedad cardiovascular que asiste al programa de rehabilitación cardiaca. Investigación en Enfermería: Imagen y Desarrollo. 2011; 13(2): $49-74$

11. Zimmermann, González, Galán. Perfiles de exposición de riesgo cardiovascular según la ocupación laboral en la Comunidad de Madrid. Rev. Esp. Salud Publica. 2010; 84(3): 293-308. 
12. Burgos P, Ruiz T, Queipo D, Rescalvo F, Martínez M, Amo P. Calidad de vida relacionada con la salud en trabajadores sanitarios. Med. Segur. Trab. 2012; 58(226): 27-34.

13. Ceballos P, Valenzuela S, Paravic T. Factores de riesgo psicosociales en el trabajo: género y enfermería. Av Enferm. 2014; 32(2):271-79.

14. Cassani R, Nobre F, Pazin F, Schmidt A. Prevalencia de factores de riesgo cardiovascular en trabajadores de una industria brasileña. Arq. Bras. Cardiol. 2009; 92(1): 16-22.

15. Alonso JA, Calleja AB, Borbolla S. Prevalencia de los factores de riesgo cardiovascular en trabajadores de una planta metalúrgica. Med Segur Trab. 2012; 58 (228) 269-281.

16. Consejo Internacional de Enfermeras. Las enfermeras: Una fuerza para el cambio Un recurso vital para la salud. [Acceso 14 agosto 2014]. 2014. Disponible en: http://www.icn.ch/images/stories/documents/ publications/ind/IND_Kit_2014_SP.pdf

17. Hernández WI. Factores de riesgo de enfermedad cardiovascular en una población obrera industrial de la provincia de Cartago. Rev. costarric. salud pública. 2000; 9(16): 55-64.

18. Quirós M, Delgadillo B. Estimación del Riesgo Cardiovascular en la Población Atendida en Consulta Externa del Área de Salud de Ciudad Quesada, durante los Años 2008 y 2009. Revista Médica de Costa Rica y Centroamérica. 2012; LXIX (604): 533-538.
19. Organización Mundial de la Salud. Cerrando la Brecha: La Política de Acción sobre los Determinantes Sociales de la Salud. Río de Janeiro [Brasil]: Conferencia Mundial sobre los Determinantes Sociales de la Salud; 2011.

20. Vidal D, Chamblas I, Zavala M, Müller R, Rodríguez MC, Chávez A Determinantes Sociales en Salud y Estilos de Vida en Población Adulta de Concepción, Chile. Cienc. Enferm. 2014; 20(1): 61-74.

21. Nitsche MP, Bitran M, Pedrals N, Echeverría G, Rigotti A. Recursos psicosociales positivos y salud cardiovascular. Rev Med Chile. 2014; 142 : 1317-1318.

22. Guerrero JM, Sánchez JG. Factores protectores, estilos de vida saludable y riesgo cardiovascular. Psicología y Salud. 2015; 25(1): 63-64.

23. Tengland PA. Health Promotion and Disease Prevention: Logically Different Conceptions? Health Care Anal. 2010; 18:323-41.

24. Herrera E, Céspedes VM, Flores ML. La enfermería y el cuidado para la salud cardiovascular: análisis de concepto. av.enferm. 2014; XXXII (1): 147-53.

25. Pérez-Noriega $E$, Soriano-Sotomayor MM, Lozano-Galindo $V$, Morales ML, de la Luz LM, Rugerio MA. Factores de riesgo cardiovascular en población adulta aparentemente sana de la ciudad de Puebla. Revista Mexicana de Cardiología. 2008; 16(3): 87-92.

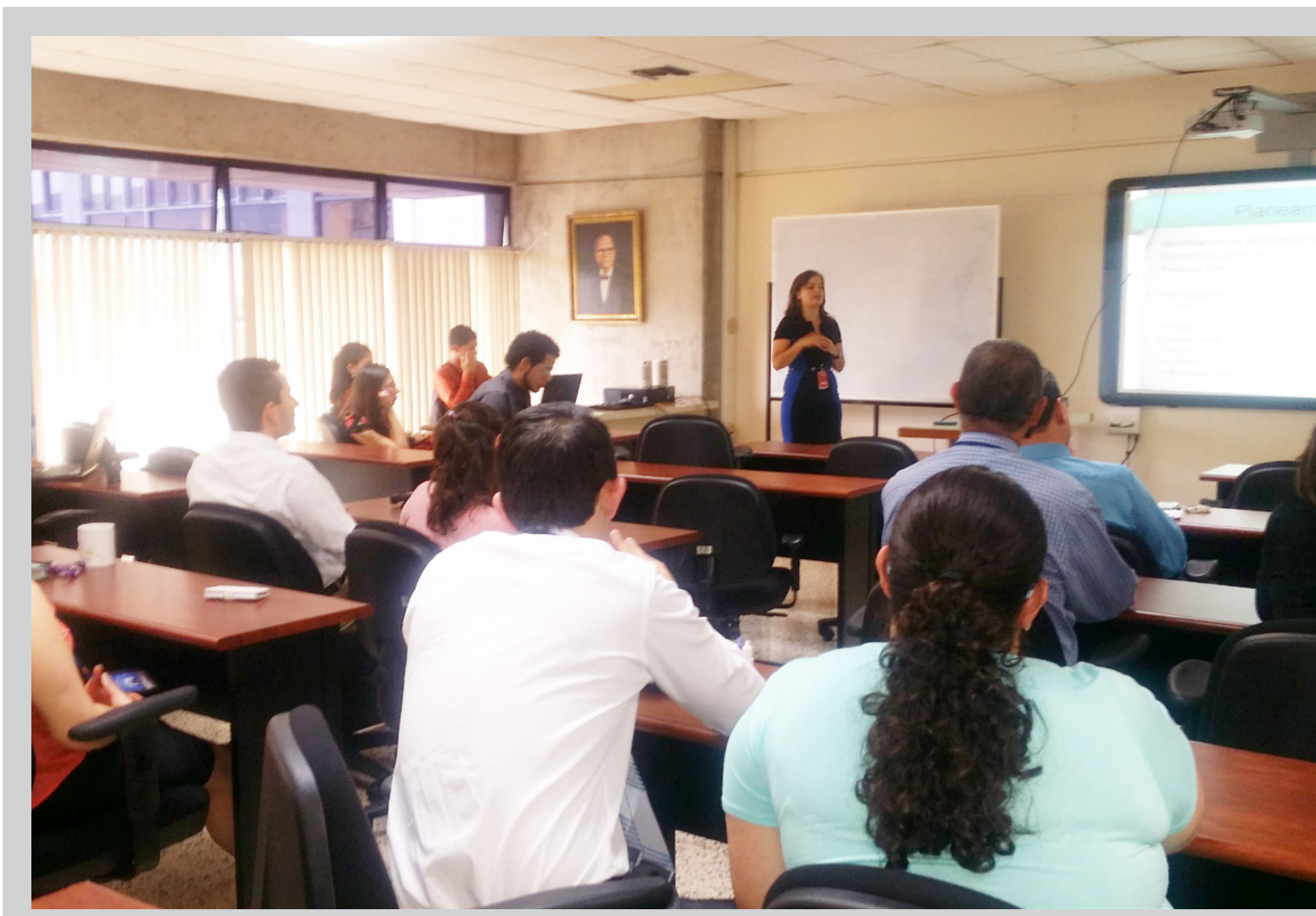


26. Lira MT, Kunstmann S, Caballero E, Guarda E, Villarroel L, Molina JC. Prevención cardiovascular y actitud de cambio frente a los factores de riesgo: un análisis crítico del estado actual. Rev. méd. Chile. 2006; 134(2): 223-30.

27. Nanda Internacional. Diagnósticos enfermeros: definiciones y clasificación, 2012-2014. Barcelona [España]: Elsevier; 2013.

28. Botija MP, Lizán L, Gosalbes V, Bonet A, Fornos A. How does intensive therapy to control cardiovascular risk factors affect health-related quality of life in diabetic patients? Aten Primaria. 2007; 39(5):227-233.

29. Gordon H, Guyatt MD. Measurement of Health-Related Quality of Life in Heart Failure. JACC. 1993; 22 (4) (Suplemento A): 185A-91A.

30. Lizán L. La calidad de vida relacionada con la salud. Revista Atención Primaria. 2009; 41(7):411-16.

31. Soto M, Failde I. La calidad de vida relacionada con la salud como medida de resultados en pacientes con cardiopatía isquémica. Rev Soc Esp Dolor 2004; 11: 505-514

32. Schwartzmann L. Calidad de vida relacionada con la salud: aspectos conceptuales. Cienc Enferm. 2003; 9(2): 09-21.

33. Blokstra A, van Dis I, Verschuren M. Efficacy of multifactorial lifestyle interventions in patients with established cardiovascular diseases and high risk groups. Eur J Cardiovasc Nurs. 2012; 11(1): 97-104
34. Vílchez-Barboza V, Paravic T, Salazar A, Sáez K. Efecto de Intervención Innovadora: Consejería de Enfermería en Salud Cardiovascular en Atención Primaria. Rev Chil Cardiol. 2015; 34:36-44.

35. Casas SB, Klijn TP. Pde la salud y su entorno laboral saludable. Rev Latino-am Enfermagem. 2006; 14(1):136-41.

36. Fawcett J. Contemporary Nursing Knowledge. Analysis and Evaluation of Nursing Models and Theories. 2ed. Philadelphia: F.A. Davis Company; 2005

37. Kerouac S, Pepin J, Ducharme F, Duquette A, Major F. El pensamiento enfermero. España: Elsevier Masson; 2007.

38. Newman MA, Sime AM, Corcoran-Perry SA. The focus of the discipline of nursing. Adv Nurs Sci. 1991; 14(1): 1-6.

39. Bueno S. Aspectos Ontológicos y Epistemológicos de las Visiones de Enfermería Inmersas en el Quehacer Profesional. Cienc Enferm. 2011; XVII (1): 37-43.

40. Marriner A, Raile M. Modelos y teorías de enfermería.7ed. Barcelona - España: Elsevier. 2007.

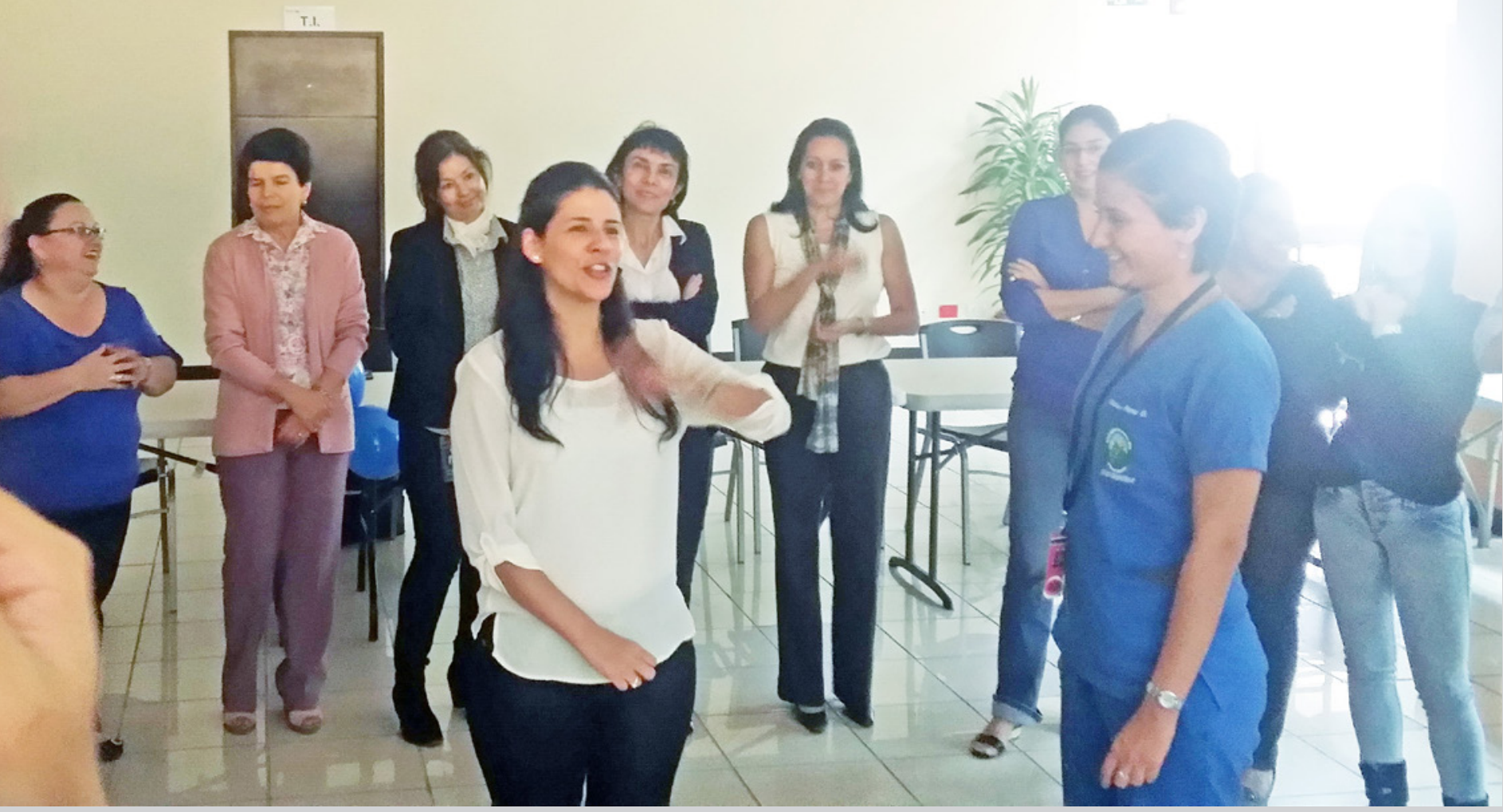

